\title{
Оптимизация диэлектрического волновода для лазерных структур дальнего инфракрасного диапазона на основе $\mathrm{HgTe} / \mathrm{CdHgTe}$
}

\author{
(C) А.А. Дубинов ${ }^{1,2}$, В.В. Румянцев ${ }^{1,2}$, М.A. Фадеев ${ }^{1}$, В.В. Уточкин ${ }^{1}$, С.В. Морозов ${ }^{1,2, \text { Ф }}$ \\ ${ }^{1}$ Институт фризики микроструктур Российской академии наук, \\ 603950 Нижний Новгород, Россия \\ ${ }^{2}$ Нижегородский государственный университет им. Н.И. Лобачевского, \\ 603950 Нижний Новгород, Россия \\ ฯ E-mail: more@ipmras.ru
}

Поступила в Редакцию 22 декабря 2020 г.

В окончательной редакции 30 декабря 2020 г.

Принята к публикации 30 декабря 2020 г.

\begin{abstract}
Проведена оптимизация параметров диэлектрического волновода для лазерных гетероструктур с квантовыми ямами на основе $\mathrm{HgCdTe}$, рассчитанных на диапазон длин волн 25-41 мкм, с точки зрения минимизации внутренних потерь. Показано, что для излучения в диапазоне длин волн 25-33.5 мкм оптимальный вариант волновода реализуется при росте лазерной $\mathrm{HgCdTe-структуры} \mathrm{на} \mathrm{подложке} \mathrm{CdTe} \mathrm{или} \mathrm{на} \mathrm{подложке} \mathrm{GaAs} \mathrm{c}$ толстым (15 мкм и более) буферным слоем CdTe. Для более длинноволнового излучения (диапазон длин волн 33.5-41 мкм) оптимальным решением является стравливание подложки и буферного слоя CdTe c последующей металлизацией поверхности структуры.
\end{abstract}

Ключевые слова: лазерная структура, волновод, дальний инфракрасный диапазон длин волн, $\mathrm{HgCdTe}$, квантовая яма.

DOI: 10.21883/FTP.2021.05.50837.9581

\section{1. Введение}

В настоящее время наиболее распространенными из компактных источников когерентного излучения в дальнем инфракрасном (ИК) диапазоне являются квантовокаскадные лазеры (ККЛ) на основе полупроводников $\mathrm{A}^{\mathrm{III}} \mathrm{B}^{\mathrm{V}}[1]$. Практическая потребность в подобных когерентных источниках во многом связана с актуальными задачами спектроскопии и анализа сложных соединений, в том числе органических [2], представляющих большой интерес в области биоинженерии. В то же время существует широкий диапазон длин волн (25-50 мкм), в котором ККЛ на основе $\mathrm{A}^{\mathrm{III}} \mathrm{B}^{\mathrm{V}}$ не могут работать вследствие сильного фононного поглощения этих полупроводников [3]. Альтернативу материалам $\mathrm{A}^{\mathrm{III}} \mathrm{B}^{\mathrm{V}}$ могут составить полупроводниковые системы, в которых энергии полярных оптических фононов находятся за пределами целевого спектрального диапазона; в частности, тройные соединения в системе кадмий-ртуть-теллур (КРТ, HgCdTe) имеют характерные энергии оптических фононов $\sim 17$ мэВ (длина волны $\sim 70$ мкм). Широко применяемый для создания фотоприемников и приемных матриц среднего ИК диапазона (см., например, работу [4] и ссылки в ней), этот материал в последнее время активно рассматривается и как усиливающая среда для построения длинноволновых источников излучения. Так, недавно было предложено использовать структуры с квантовыми ямами (КЯ) на основе $\mathrm{HgCdTe}$ для создания ККЛ, излучающего на длине волны 36 мкм [5]. Недостатком данного подхода, ограничивающим гибкость решений на основе ККЛ в задачах спектроскопии, можно назвать скромные возможности перестройки длины волны излучения таких источников (в частности, за счет изменения температуры излучателя), что обусловлено межподзонным характером оптических переходов в ККЛ. С этой точки зрения возможность реализации в структурах на основе $\mathrm{HgCdTe}$ усиления в дальнем ИК диапазоне на межзонных оптических переходах является несомненно интересной, и здесь необходимо отметить широчайший диапазон длин волн, потенциально доступный для структур на основе $\mathrm{HgCdTe}$ при варьировании состава тройного раствора, - от 0 до 1.6 эВ [4].

Концепция межзонных лазеров на основе пленок $\mathrm{HgCdTe,} \mathrm{излучающих} \mathrm{в} \mathrm{среднем} \mathrm{ИК} \mathrm{диапазоне} \mathrm{при}$ оптической накачке, далеко не нова (см., например, [6]). В то же время лишь сравнительно недавно прогресс в области эпитаксиального роста КРТ-структур позволил уверенно формировать гетероструктуры с квантовыми ямами (КЯ) $\mathrm{HgTe} / \mathrm{HgCdTe}$. Такие структуры, обеспечивающие уникальные возможности управления зонным спектром носителей заряда, определили новые возможности развития лазерных структур. На настоящий момент структуры с КЯ $\mathrm{HgTe} / \mathrm{HgCdTe}$ позволили получить стимулированное излучение (СИ) при оптической накачке на длинах волн до 20 мкм [7]. Теоретические же оценки показывают, что генерация СИ возможна на длинах волн до 60 мкм при криогенных температурах [8]. Следует, однако, уточнить, что в [8] оценены фактически пороги прозрачности КЯ, но не обсуждаются какиелибо потери в пассивных (волноводных) слоях, имеющие место в реальных лазерных структурах. При этом, если поглощением в нелегированных волноводных слоях по механизму Друде можно зачастую пренебречь, то фононное поглощение в растворах $\mathrm{HgCdTe}$ в рассматри- 
Параметры слоев лазерных структур для рассматриваемых диэлектрических волноводов

\begin{tabular}{|c|c|c|c|c|c|c|}
\hline Слои & $\begin{array}{l}\text { Волновод } \\
\quad \# 1\end{array}$ & $\begin{array}{l}\text { Волновод } \\
\quad \# 2\end{array}$ & $\begin{array}{l}\text { Волновод } \\
\text { \#3 }\end{array}$ & $\begin{array}{l}\text { Волновод } \\
\quad \# 4\end{array}$ & $\begin{array}{l}\text { Волновод } \\
\quad \# 5\end{array}$ & $\begin{array}{l}\text { Волновод } \\
\quad \# 6\end{array}$ \\
\hline Подложка & GaAs & GaAs & GaAs & $n^{+}-\mathrm{GaAs}$ & CdTe & Стравливается \\
\hline $\begin{array}{l}\text { Буфер, } \\
\text { толщина }\end{array}$ & $\begin{array}{l}\text { CdTe, } \\
5 \text { мкм }\end{array}$ & $\begin{array}{l}\text { CdTe, } \\
10 \text { мкм }\end{array}$ & $\begin{array}{l}\text { CdTe, } \\
15 \text { мкм }\end{array}$ & $\begin{array}{l}\text { CdTe, } \\
5 \text { мкм }\end{array}$ & CdTe, & Стравливается \\
\hline $\begin{array}{l}\text { Волноводный слой, } \\
\text { толщина }\end{array}$ & $\begin{array}{l}\mathrm{HgCdTe}, \\
8.5 \text { мкм }\end{array}$ & $\begin{array}{l}\mathrm{HgCdTe}, \\
4.5 \text { мкм }\end{array}$ & $\begin{array}{l}\mathrm{HgCdTe}, \\
3 \text { мкм }\end{array}$ & $\begin{array}{l}\mathrm{HgCdTe}, \\
2.5 \text { мкм }\end{array}$ & $\begin{array}{l}\mathrm{HgCdTe}, \\
2.5 \text { мкм }\end{array}$ & $\begin{array}{l}\mathrm{HgCdTe}, \\
2 \text { мкм }\end{array}$ \\
\hline $\begin{array}{l}20 \text { КЯ с барьерами, } \\
\text { толщина }\end{array}$ & $\begin{array}{c}\mathrm{HgTe} / \mathrm{HgCdTe}, \\
0.42 \text { мкм }\end{array}$ & $\begin{array}{c}\mathrm{HgTe} / \mathrm{HgCdTe}, \\
0.42 \text { мкм }\end{array}$ & $\begin{array}{c}\mathrm{HgTe} / \mathrm{HgCdTe}, \\
0.42 \text { мкм }\end{array}$ & $\begin{array}{c}\mathrm{HgTe} / \mathrm{HgCdTe}, \\
0.42 \text { мкм }\end{array}$ & $\begin{array}{c}\mathrm{HgTe} / \mathrm{HgCdTe}, \\
0.42 \text { мкм }\end{array}$ & $\begin{array}{c}\mathrm{HgTe} / \mathrm{HgCdTe}, \\
0.42 \text { мкм }\end{array}$ \\
\hline $\begin{array}{l}\text { Волноводный слой, } \\
\text { толщина }\end{array}$ & $\begin{array}{c}\text { HgCdTe, } \\
9.5 \text { мкм }\end{array}$ & $\begin{array}{l}\mathrm{HgCdTe}, \\
6 \text { мкм }\end{array}$ & $\begin{array}{l}\mathrm{HgCdTe} \\
5 \text { мкм }\end{array}$ & $\begin{array}{l}\mathrm{HgCdTe}, \\
3.75 \text { мкм }\end{array}$ & $\begin{array}{c}\mathrm{HgCdTe} \\
4.5 \text { мкм }\end{array}$ & $\begin{array}{l}\mathrm{HgCdTe} \\
5 \text { мкм }\end{array}$ \\
\hline Покровный слой & $\begin{array}{c}\text { CdTe, } \\
0.05 \text { мкм }\end{array}$ & $\begin{array}{c}\text { CdTe, } \\
0.05 \text { мкм }\end{array}$ & $\begin{array}{c}\text { CdTe, } \\
0.05 \text { мкм }\end{array}$ & $\begin{array}{c}\text { CdTe, } \\
0.05 \text { мкм }\end{array}$ & $\begin{array}{c}\text { CdTe, } \\
0.05 \text { мкм }\end{array}$ & $\begin{array}{c}\text { CdTe, } \\
0.05 \text { мкм }\end{array}$ \\
\hline Пограничный слой & Вакуум & Вакуум & Вакуум & Вакуум & Вакуум & $\mathrm{Au}$ \\
\hline
\end{tabular}

ваемом диапазоне частот является существенным. Кроме того, в зависимости от конкретных параметров волновода важным может оказаться и учет фононного поглощения в подложках $\mathrm{GaAs}$, часто использующихся для выращивания лазерных структур на основе $\mathrm{HgCdTe}$ [7].

В данной работе сопоставлен ряд возможных вариантов волноводов лазерных структур на основе $\mathrm{HgCdTe}$, для чего вычислены коэффициенты поглощения излучения в пассивных слоях структуры и коэффициент усиления для волноводной моды с учетом достижимого фактора оптического ограничения.

\section{2. Результаты расчетов и обсуждение}

Базовым условием развития лазерной генерации является превышение величины усиления над общими потерями в структуре, что может быть выражено в виде

$$
\left(G-\alpha_{a}\right) \Gamma=\alpha_{p}+\alpha_{m},
$$

где $G$ - коэффициент усиления активной среды, $\alpha_{a}-$ коэффициент потерь в активной среде, $\Gamma$ - фактор оптического ограничения, $\alpha_{p}$ и $\alpha_{m}-$ коэффициенты поглощения в пассивных слоях и потерь на зеркалах соответственно. Расчеты, приведенные в [8], касаются случая $G=\alpha_{a}$ (без учета $\alpha_{p}$ и $\alpha_{m}$ ). Целью данной работы является учет внутренних потерь в волноводе $\left(\alpha_{p}\right)$, в то же время мы ограничимся рассмотрением режима суперлюминесценции (однопроходного усиления излучения), пренебрегая потерями на зеркалах $\alpha_{m}$.

Для анализа были выбраны шесть различных волноводов для лазерных структур на основе $\mathrm{HgCdTe}$; конкретные параметры слоев для каждого из волноводов приведены в таблице. Первые три варианта волновода предполагают рост на полуизолирующей подложке $\mathrm{GaAs}$ с буферным слоем из нелегированного CdTe и различаются соотношением толщин буферного (CdTe) и ограничивающих (CdHgTe) слоев, четвертый рассчитан для роста на сильно легированной подложке $\mathrm{GaAs}$, пятый - на нелегированной подложке $\mathrm{CdTe}$. Наконец, последний вариант предполагает стравливание подложки и буферного слоя с последующим напылением слоя золота, подобный метод используется при создании ККЛ с двойным металлическим волноводом [9]. Для всех структур состав ограничивающих/барьерных слоев $\mathrm{Hg}_{1-x} \mathrm{Cd}_{x} \mathrm{Te}$ был фиксированным $(x=0.75)$. Активная область во всех рассматриваемых случаях содержала 20 КЯ HgTe/CdHgTe.

Оптимизация лазерных структур проводилась для генерации основной моды $\mathrm{TE}_{0}$. Действительно, в КЯ $\mathrm{HgTe} / \mathrm{HgCdTe}$ основной дырочный уровень представлен состояниями тяжелых дырок, и в этом случае, согласно правилам отбора для переходов электронов между валентной зоной и зоной проводимости [10], усиление возможно только для таких электромагнитных мод волновода, в которых компонента электрического поля лежит в плоскости КЯ. В нашем случае такой компонентой электрического поля обладают только ТЕ-моды. Для нахождения распределения электрического поля ТЕ-моды, определения эффективного показателя преломления и коэффициента поглощения $\alpha_{p}$ для волноводных мод проводилось численное решение уравнений Максвелла $[11,12]$. Диэлектрические проницаемости нелегированных GaAs и CdTe, а также $\mathrm{Au}$ аппроксимировались на основании экспериментальных данных, представленных в [13]. В отсутствие экспериментальных данных по частотной зависимости диэлектрической проницаемости слоев $\mathrm{Hg}_{0.25} \mathrm{Cd}_{0.75} \mathrm{Te}$ в интересующей нас области спектра (на длинах волн 25-41 мкм) было использовано приближение, в котором действительная часть диэлектрической проницаемости принималась равной 8.1 [4], а мнимая часть считалась такой же, как в CdTe. Чтобы учесть влияние легирования в подложке GaAs, к значению диэлектрической проницаемости 


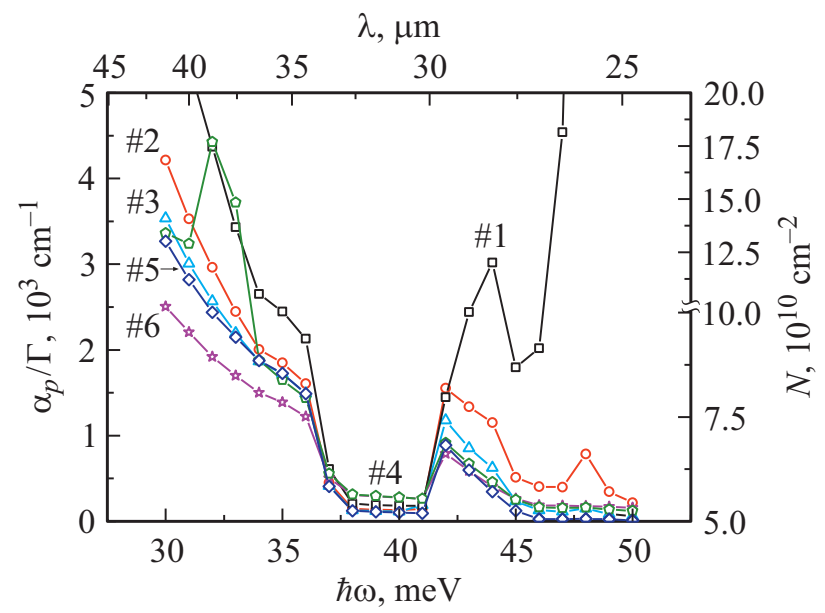

Рис. 1. Зависимости $\alpha_{p} / \Gamma$ и необходимой для достижения этой величины концентрации фотовозбужденных носителей $N$ от энергии кванта и длины волны излучения для всех 6 конструкций волноводов. Указаны номера волноводов.

нелегированного GaAs добавлялась соответствующая „проводящая“ часть [14]

$$
\Delta \varepsilon(\omega)=-\frac{\omega_{p}^{2} \varepsilon_{\infty}}{\omega^{2}+i \gamma \omega}
$$

где $\omega_{p}^{2}=4 \pi N_{e} q^{2} /\left(m^{*} \varepsilon_{\infty}\right)-$ квадрат плазменной частоты, $\gamma=q / m^{*} \mu-$ фактор потерь, $q-$ заряд электрона, $\mu$ и $m^{*}$ - подвижность и эффективная масса электронов соответственно, $N_{e}$ - концентрация свободных электронов (считалась равной $2 \cdot 10^{18} \mathrm{~cm}^{-3}$ ). Использовались данные справочника [15] относительно подвижности $\mu$ электронов при таком уровне легирования GaAs, а также величин $\varepsilon_{\infty}$ и $m^{*}$.

На рис. 1 представлены величины потерь $\alpha_{p} / \Gamma$, вычисленные для всех рассматриваемых волноводов в зависимости от энергии кванта $\hbar \omega$ (длины волны излучения $\lambda$ ). Согласно (1), минимум величины $\alpha_{p} / \Gamma$ соответствует минимальному коэффициенту усиления $G$ в КЯ, необходимому для возникновения стимулированного излучения. Для рассматриваемых структур толщины волноводных слоев подбирали исходя из условия минимальной величины $\alpha_{p} / \Gamma$ при энергии кванта 35 мэВ, соответствующей длине волны излучения $\lambda=35.4$ мкм. Необходимо отметить две характерные особенности в полученных зависимостях величины $\alpha_{p} / \Gamma$ от длины волны излучения, наблюдаемые для всех вариантов волноводов. Первая - резкое увеличение потерь $\alpha_{p} / \Gamma$ на длинах волн $\lambda>33.5$ мкм, что обусловлено резким ростом с длиной волны $\alpha_{p}$ в $\mathrm{CdTe}$ и $\mathrm{Hg}_{0.25} \mathrm{Cd}_{0.75} \mathrm{Te}$, связанное с возрастанием вклада однофононного поглощения в этих материалах по мере продвижения в область длинных волн. Вторая особенность - выраженный пик потерь в диапазоне длин волн 27.5-29.5 мкм, определяемый уже двухфононным поглощением в этих материалах.
Из рис. 1 видно, что существует диапазон длин волн 29.5-33.5 мкм, в котором все рассматриваемые волноводы имеют минимальные (и относительно близкие по величине) значения величины потерь. Заметим, что на длинах волн в диапазоне 25-33.5 мкм оптимальным был бы вариант \#5 (лазерная структура, выращенная на подложке $\mathrm{CdTe}$ ). В то же время подложки $\mathrm{CdTe}$ достаточно редки и более практичным выбором здесь представляются варианты волновода, сформированного на полуизолирующей подложке GaAs c толстым (> 15 мкм) буферным слоем CdTe (\#3 в таблице) либо на сильно легированной подложке $\mathrm{GaAs}(\# 4)$. Также достаточно эффективным в этом диапазоне будет металлизированный волновод (\#6), волновод с относительно тонким CdTe-буфером (\#1) будет хорошо работать лишь в узком интервале длин волн 29.5-33.5 мкм. Для более длинноволнового излучения (на длине волны в пределах 33.5-41 мкм) оптимальным будет металлизированный волновод (\#6).

При сопоставлении пассивных потерь в волноводах различной конструкции (рис. 1) мы ограничили рассматриваемый диапазон потерь на уровне $\left(\alpha_{p} / \Gamma\right)_{\max }<5 \cdot 10^{3} \mathrm{~cm}^{-1}$, что приблизительно соответствует величине усиления, достижимой в диапазоне длин волн 25-41 мкм в одиночной КЯ $\mathrm{HgTe} / \mathrm{HgCdTe}$. На рис. 2 приведен соответствующий расчет коэффициента усиления $\left(G-\alpha_{a}\right)$ в зависимости от концентрации фотовозбужденных носителей $N$, проведенный в соответствии с методикой [16] (температура решетки полагается равной $T_{0}=4.2 \mathrm{~K}$, температура носителей $\left.T_{e}=77 \mathrm{~K}\right)$. Видно, что просветление КЯ $\left(G=\alpha_{a}\right)$ наступает при пороговой концентрации носителей $N_{\mathrm{th}} \approx 5 \cdot 10^{10} \mathrm{~cm}^{-2}$, после чего усиление сублинейно растет с увеличением $N$ и достигает $\left(G-\alpha_{a}\right)_{\max } \approx 5 \cdot 10^{3} \mathrm{~cm}^{-1}$ при $N_{\max } \approx 2 \cdot 10^{11} \mathrm{~cm}^{-2}$. Указанная концентрация носителей $N_{\max }$ является, по-видимому, максимальной, peа-

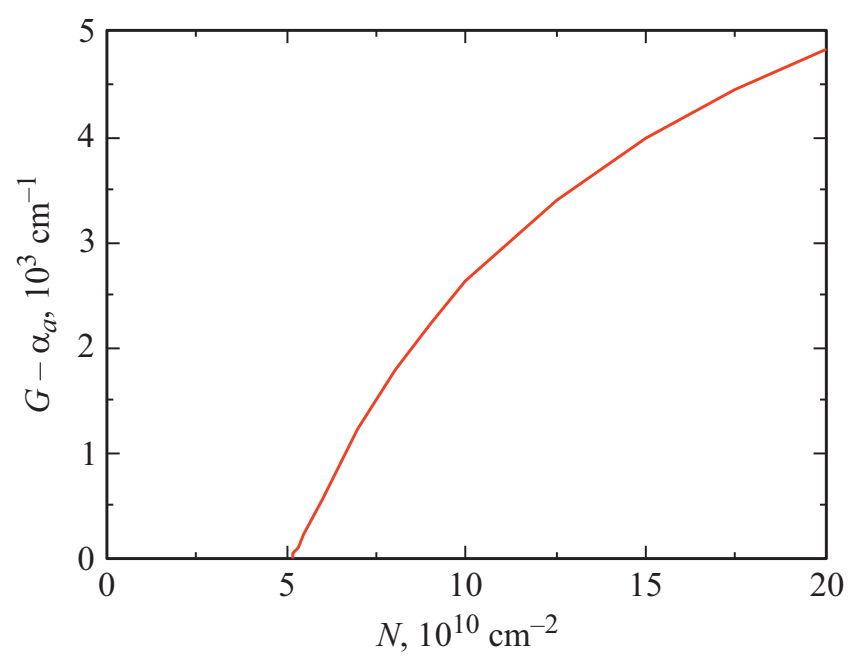

Рис. 2. Зависимость коэффициента усиления в КЯ $\left(G-\alpha_{a}\right)$ от концентрации фотовозбужденных носителей $N$ в КЯ $\mathrm{HgTe} / \mathrm{HgCdTe}$ для диапазона длин волн 25-40 мкм. 
лизуемой в „длинноволновых“ КЯ $\mathrm{HgTe} / \mathrm{HgCdTe}$ при разумных мощностях накачки. Так, при меньших концентрациях носителей в КЯ $\mathrm{HgTe} / \mathrm{HgCdTe}$ преобладающим процессом межзонной рекомбинации является оже-рекомбинация [17]. Характерные времена этого процесса - субнаносекундные, $\tau \approx 0.5 \mathrm{Hc}$ при $N_{\text {th }} \approx 5 \cdot 10^{10} \mathrm{~cm}^{-2}$ и $\sim 0.1$ нс при $N_{\max } \approx 2 \cdot 10^{11} \mathrm{~cm}^{-2}$. При превышении величины $N_{\max }$ пороговым образом включается сверхбыстрый процесс межзонной рекомбинации (времена $\sim 1$ пс), сопровождающийся генерацией двумерных плазмонов [17]. За счет этого процесса мощность накачки, необходимая для достижения схожей концентрации носителей, возрастает на 2 порядка величины. Отметим, что при этом в принципе возможна стимулированная генерация плазмонов, сильно локализованных вблизи КЯ и не требующих дополнительного волновода [18]; подобные эффекты остаются за рамками данной работы.

Сопоставление расчетных данных для величин усиления (рис. 2) и потерь (рис. 1) в рассматриваемых структурах позволяет оценить пороговые плотности мощности накачки, необходимые для возникновения СИ, исходя из соотношения

$$
I_{\mathrm{th}}=\hbar \Omega N / \eta \tau \text {. }
$$

Здесь $\hbar \Omega$ и $\eta-$ энергия фотона для излучения накачки и доля излучения накачки, поглощаемого в КЯ соответственно, $\tau$ - время межзонной релаксации (оже-рекомбинации, см. выше). Если предположить, что оптическая накачка будет осуществляться $\mathrm{CO}_{2}$-лазером на длине волны 10.6 мкм ( $\hbar \Omega=117$ мэВ), а $\eta \approx 0.005$ для КЯ, для $N \approx 5 \cdot 10^{10} \mathrm{~cm}^{-2}$ порог СИ оценивается на уровне $I_{\text {th }} \approx 400 \mathrm{BT} / \mathrm{cm}^{2}$, в то время как для $N \approx 2 \cdot 10^{11} \mathrm{~cm}^{2}$ величина составит $I_{\text {th }} \approx 7.5 \mathrm{\kappa BT} / \mathrm{cm}^{2}$.

\section{3. Заключение}

В работе проведен расчет характеристик шести вариантов диэлектрического волновода лазерных структур на основе 20 КЯ $\mathrm{HgTe} / \mathrm{HgCdTe}$, излучающих в дальнем ИКдиапазоне: коэффициента поглощения, фактора оптического ограничения и усиления. Показано, что для каждой длины волны в диапазоне 25-41 мкм может быть подобран оптимальный вариант волновода, обеспечивающий возможность генерации стимулированного излучения.

\section{Финансирование работы}

Работа выполнена при поддержке Российского фонда фундаментальных исследований (проект № 20-52-50004 ЯФ_а) в части расчета внутренних (фононных) потерь в рассматриваемых диэлектрических волноводах и при поддержке Министерства образования и науки Российской Федерации (проект МК-1430.2020.2) в части расчета достижимых величин усиления в КЯ $\mathrm{HgTe} / \mathrm{CdHgTe}$.

\section{Конфликт интересов}

Авторы заявляют, что у них нет конфликта интересов.

\section{Список литературы}

[1] M.S. Vitiello, G. Scalari, B. Williams, P. De Natale. Opt. Express, 23, 5167 (2015).

[2] R.J. Falconer, A.G. Markelz. J. Infrared Milli. Terahz. Waves, 33, 973 (2012).

[3] F. Castellano, A. Bismuto, M.I. Amanti, R. Terazzi, M. Beck, S. Blaser, A. Baechle, J. Faist. J. Appl. Phys., 109, 102407 (2011).

[4] A. Rogalski. Rep. Progr. Phys., 68, 2267 (2005).

[5] D. Ushakov, A. Afonenko, R. Khabibullin, D. Ponomarev, V. Aleshkin, S. Morozov, A. Dubinov. Opt. Express, 28, 25371 (2020).

[6] I. Melngailis, A. Strauss. Appl. Phys. Lett., 8, 179 (1966).

[7] S.V. Morozov, V.V. Rumyantsev, M.A. Fadeev, M.S. Zholudev, K.E. Kudryavtsev, A.V. Antonov, A.M. Kadykov, A.A. Dubinov, N.N. Mikhailov, S.A. Dvoretsky, V.I. Gavrilenko. Appl. Phys. Lett., 111, 192101 (2017).

[8] G. Alymov, V. Rumyantsev, S. Morozov, V. Gavrilenko, V. Aleshkin, D. Svintsov. ACS Photonics, 7, 98 (2020).

[9] K. Unterrainer, R. Colombelli, C. Gmachl, F. Capasso, H. Hwang, A. Sergent, D. Sivco, A. Cho. Appl. Phys. Lett., 80, 3060 (2002).

[10] F. Bachmann, P. Loosen, R. Poprawe. High power diode lasers. Technology and applications (N. Y., Springer, 2007).

[11] Л.Д. Ландау, Е.М. Лифшиц. Электродинамика сплошных сред (М., Наука, 1989).

[12] H.C. Casey, M.B. Panich. Heterostructure lasers (N. Y., Academic Press, 1978).

[13] E.D. Palik. Handbook of optical constants of solids (Orlando, Academic Press, 1985).

[14] J.S. Blackmore. J. Appl. Phys., 53, R123 (1982).

[15] A. Dargys, J. Kundrotas. Handbook on Physical Properties of Ge, Si, GaAs and InP (Vilnius, Science and Encyclopedia Publishers, 1994).

[16] V.Ya. Aleshkin, A.A. Dubinov, V.V. Rumyantsev, M.A. Fadeev, O.L. Domnina, N.N. Mikhailov, S.A. Dvoretsky, F. Teppe, V.I. Gavrilenko, S.V. Morozov. J. Phys.: Condens. Matter, 30, 495301 (2018).

[17] V. Aleshkin, G. Alymov, A. Dubinov, V. Gavrilenko, F. Teppe. J. Phys. Commun., 4, 115012 (2020).

[18] K. Kapralov, G. Alymov, D. Svintsov, A. Dubinov. J. Phys.: Condens. Matter, 32, 065301 (2020).

Редактор Л.В. Шаронова 


\title{
Dielectric waveguide optimization \\ for the laser structures with $\mathrm{HgCdTe}$ QWs emitting in far-infrared range
}

\author{
A.A. Dubinov ${ }^{\mathbf{1}, 2}$, V.V. Rumyantsev ${ }^{\mathbf{1}, 2}$, M.A. Fadeev ${ }^{1}$, \\ V.V. Utochkin ${ }^{1}$, S.V. Morozov ${ }^{\mathbf{1}, 2}$ \\ ${ }^{1}$ Institute for Physics of Microstructures, \\ Russian Academy of Sciences, \\ 603950 Nizhny Novgorod, Russia \\ ${ }^{2}$ Lobachevsky State University of Nizhny Novgorod, \\ 603950 Nizhny Novgorod, Russia
}

\begin{abstract}
We investigate optimized designs of dielectric waveguides for $\mathrm{HgTe} / \mathrm{HgCdTe}$ quantum well heterostructures emitting in the wavelength range of $25-41 \mu \mathrm{m}$. We demonstrate that $\mathrm{HgCdTe}$-based waveguides grown directly on CdTe substrates or on GaAs substrates with thick $(\sim 15 \mu \mathrm{m})$ CdTe buffers are best suited for the $25-33.5 \mu \mathrm{m}$ spectral range, while substrate removal followed by surface metallization provides an optimal way to define a waveguide for longer wavelength emission, at $33.5-41 \mu \mathrm{m}$.
\end{abstract}

